\title{
Descifrando el currículum a través de las TIC: una visión interactiva sobre las competencias digitales de los estudiantes de Ciencias del Deporte y de la Actividad Física
}

Elena Conde Pascual

Universidad Católica San Antonio, Murcia (UCAM, España)

José Jesús Trujillo Vargas

Universidad Católica San Antonio, Murcia (UCAM, España)

Hernando Castaño Buitrago

Universitaria del Caribe (CECAR). Sincelejo (Colombia) 

Descifrando el currículum a través de las TIG: una visión interactiva sobre las competencias digitales de los estudiantes de Giencias del Deporte y de la Actividad Física

\title{
Deciphering the curriculum through ICT: an interactive vision on the digital competences of students of Sports Science and Physical Activity
}

\author{
Elena Conde Pascual \\ Facultad de Deporte, Universidad Católica San Antonio, Murcia (UCAM, España) \\ econde@ucam.edu.co
}

\section{José Jesús Trujillo Vargas}

Departamento de Educación, Universidad Católica San Antonio, Murcia (UCAM, España)

jjtrujillo@ucam.edu

\section{Hernando Gastaño Buitrago}

Facultad de Ciencias Económicas y Administrativas de la Corporación Universitaria del Caribe (CECAR). Sincelejo (Colombia) hernando.castano.edu.co

Fecha de recepción: 12/01/2017

Fecha de aceptación: 20/04/2017

\section{Resumen}

El conocimiento y dominio en la sociedad actual de las nuevas tecnologías de la información y la Comunicación (TIC), se ha convertido en un elemento de relevancia en el ámbito académico y especialmente en el marco universitario, donde su utilización permite, entre otros, el desarrollo de nuevas estrategias metodológicas con el objetivo de la mejora el aprendizaje autónomo del alumno. El objetivo del presente estudio fue conocer el grado de conocimiento y formación sobre las competencias básicas digitales que poseen los alumnos de Ciencias del Deporte y de la Actividad Física de la Corporación Universitaria del Caribe (CECAR), para lo que se ha utilizado el cuestionario "Competencias básicas digitales 2.0 de estudiantes universitarios" COBADI 2013, adaptado a las características del español en Colombia y al entorno estudiado. El diseño del presente estudio es de tipo no experimental y la muestra final fue de 89 alumnos. Entre los resultados del estudio, cabe destacar el elevado porcentaje de alumnos que utilizan Internet, en 
mayor medida, para buscar información para tareas universitarias (78\%), realizar actividades académicas (63\%) y consultar información sobre temas académicos y profesionales.

Palabras clave: Educación; TIC; Moodle; Competencia; Profesor.

\begin{abstract}
Knowledge and domain in today's society of the new information and communications technology (ICT) has become an important element in academic and especially in university settings where its use allows, among others, development of new methodological strategies aimed at improving student learning autonomous. The aim of this study was to determine the degree of knowledge and training on digital basic skills possessed by students of Sport Science and Physical Education of the University Corporation of the Caribbean (CECAR), for which we have used the questionnaire "Basic skills of college students digital 2.0 "COBADI 2013, adapted to the characteristics of Spanish in Colombia and studied environment. The design of this study is not experimental and the final sample was 89 students. Among the findings, it highlight the high percentage of students who use the Internet, further, to find information for university tasks (78\%), carry out academic activities (63\%) and view information about academic and professional issues.
\end{abstract}

Keywords: Education; ICT; Moodle; Competition; Professor.

Para citar este artículo: Conde Pascual. E.; Trujillo Vargas, J. J. y Castaño Buitrago, H. (2017). Descifrando el currículum a través de las TIC: una visión interactiva sobre las competencias digitales de los estudiantes de Ciencias del Deporte y de la Actividad Física.. Revista de Humanidades, n. 31, p. 195-214, ISSN 1130-5029 (ISSN-e 2340-8995).

Sumario: 1. Introducción. 2. Metodología. 3. Resultados. 4. Discusión y conclusiones. 5. Referencias bibliográficas. 6. Fuentes electrónicas.

\title{
1. INTRODUGGIÓN
}

En tanto que la característica distintiva de la primera revolución industrial parece que fue la de sustituir la energía humana o animal por la potencia mecánica, la segunda revolución industrial, también conocida como la revolución cibernética, está creando unos procesos que traen como resultado una especie de extensión de la mente humana (Blázquez, 2001: 16).

El desarrollo y rápido crecimiento de las TIC en las últimas décadas, ha provocado profundos cambios en nuestra sociedad, afectando, como no podría ser de otra manera, a la educación y a la formación en todos los niveles. Ambas se convierten en uno de los pilares fundamentales de esta nueva sociedad, con el propósito de formar a los futuros ciudadanos para esta nueva sociedad del conocimiento. Esto ha 
supuesto que las TIC se hallan ido incorporando progresivamente en todos los niveles de los diferentes sistemas educativos, propiciando una interacción, a diferentes niveles, con todos los miembros de la comunidad educativa (Almerich y otros, 2005).

El Espacio Iberoamericano de Educación Superior (EIBES) es desde hace algunos años uno de los temas más recurrentes que se mencionan en las Declaraciones de las Cumbres Iberoamericanas de Jefes de Estado y de Gobierno, de los Ministros de Educación y de los Encuentros Iberoamericanos de Rectores, en donde se muestran convencidos de la necesidad de crear un espacio académico iberoamericano de cooperación universitaria que fomente la integración de esta región, regido por los principios de autonomía universitaria, reciprocidad, solidaridad, multilateralidad, coparticipación, igualdad de oportunidades, flexibilidad, pertinencia y calidad (Blasco, 2005). El objetivo de esta política de integración de tecnologías de la información y comunicación (TIC) es lograr acercarse al nuevo paradigma educativo, como así ocurre en el EEES, inspirándose para ello en las nuevas demandas de la sociedad del conocimiento (Sánchez, Sánchez y Ramos, 2012). Para dar respuesta a este nuevo marco contexual, Esteve Mon y Gisbert (2011) plantean que las universidades deben flexibilizarse y desarrollar nuevas vías de integración de las TIC, que se ajusten a las nuevas necesidades de los estudiantes nacidos en un entorno digital (Prensky, 2001).

La importancia de educar a lo largo de la vida para responder a las necesidades y demandas sociales, recobra un nuevo sentido en la actualidad, debido a los cambios sociales, culturales y educativos que se presentan (Veytia, 2013). Nos encontramos en un momento sociohistórico muy definido, debido a la existencia y el uso continuado y generalizado de múltiples y variadas tecnologías de la información y la comunicación. Es por ello, entre otros múltiples factores, por lo que esta época reciente dista mucho de otras anteriores, donde los factores de socialización y aprendizaje eran más previsibles y homogéneos.

Los elementos que explican la enorme capacidad de cambio que presenta continuamente nuestra sociedad son entre otros: "la acumulación de información, la velocidad de su transmisión, la superación de las limitaciones o barreras espaciales, el empleo simultáneo de múltiples medios (imagen, sonido, texto, código)" (De Pablos, 2010: 7).

Numerosas investigaciones, tanto nacionales como internacionales, (Abad, 2005; Area, 2010; Cabero y Llorente, 2005, 2008; Cole y Helen, 2007; Rodríguez Damián y otros, 2009; Pérez Rodríguez y otros, 2009) resaltan el significado que deben tener las TIC en la educación superior y se centran en la definición de las competencias digitales necesarias, y de obligada adquisición, para que los individuos puedan desenvolverse de manera solvente, en base a las demandas de la sociedad contemporánea.

Más allá de la dicotomía entre nativos e inmigrantes digitales (Prensky, 2001), se presenta este estudio como una aproximación a las competencias que poseen los 
alumnos a la hora de construir su propio conocimiento y para ello, es evidente, el profesor tiene que erigirse en el mediador entre lo que ocurre a nivel social y la materia que imparte, en aras de ayudar a construir profesionales íntegros y competentes, ayudándose de herramientas como Moodle para que esto sea posible. La plataforma de formación denominada Moodle fue diseñada por Martin Dougiamas en 2002. Para el desarrollo de la plataforma se apoyó en las ideas del constructivismo pedagógico, quien afirma que el conocimiento se construye en la mente del alumnado en lugar de ser transmitido sin cambios a partir de libros o enseñanzas, y en el aprendizaje colaborativo. (Marín, Ramírez y Sampedro, 2011: 113).

Al hablar de competencias que requiere el individuo (futuro profesional) para desenvolverse de manera eficiente en la sociedad del siglo XXI, es necesario referirse a las competencias digitales, que entendemos como "el conjunto de habilidades y destrezas relacionadas con la búsqueda, selección, comprensión, análisis, síntesis, valoración, procesamiento y comunicación de información en diferentes lenguajes (verbal, numérico, icónico, visual, gráfico y sonoro), que integra conocimientos, procedimientos y actitudes" (Escamilla, 2008: 77). En este sentido, se pone el acento en la movilización articulada e interrelacionada de diferentes tipos de conocimiento, más allá de las características específicas de cada disciplina (De Pablos, 2010). Por tanto, la figura del profesor cobra una dimensión que anteriormente no poseía, ya que debe replantearse no sólo su manera de abordar el proceso de enseñanza-aprendizaje, sino también su actitud vital ante este contexto, en donde lo virtual juega un papel determinante. Las TIC ofrecen un nuevo entorno a la enseñanza, y como consecuencia un nuevo desafío al sistema educativo: pasar de un único modelo unidireccional de formación, donde por lo general los saberes residen en los profesores y profesoras, a modelos más abiertos y flexibles, donde la información tiende a ser compartida en red y centrada en los alumnos y alumnas (Palomo, Ruiz y Sánchez, 2006: 25)

Más allá de los miedos que pueda causar al profesorado el hecho de no dominar las diferentes herramientas tecnológicas, o que incluso muchos de sus alumnos tengan un conocimiento más avanzado sobre algunas de estas, los docentes han de aspirar a que este nuevo contexto se transforme en un continuo intercambio, construcción y manejo crítico del conocimiento, en donde la enseñanza tradicional y unidireccional de los contenidos no tenga cabida. Se hace importante, pues, resaltar que "la autoridad de los profesores ya no deriva de tener el monopolio del conocimiento, sino de la capacidad para enseñar a elaborar la información y a aprender"'(De Pablos, 2010: 14).

Mayor responsabilidad, si cabe, es la de los docentes de educación superior, sobre todo en los grados orientados hacia la docencia, ya que se tiene la posibilidad de ayudar a generar futuros profesores que apuesten por el uso cotidiano de las nuevas tecnologías en sus aulas escolares, a fin de que sus alumnos interpreten el mundo en el que viven y a la par, construyan una visión crítica del uso que se hace a nivel social de esos medios tecnológicos y, consecuentemente, sean capaces de llevar a cabo un uso constructivo tendente a la mejora de dicha sociedad. Relacionar el 
currículum escolar, los profesores y los alumnos, supone uno de los cruces temáticos más interesantes en el pensamiento educativo, no sólo porque pone en contacto tres componentes básicos del mismo, sino porque toca muy directamente la práctica educativa, siendo esencial considerar esta interacción para entenderla y poder cambiarla. El currículum es la partitura de la cultura escolar en contenidos y formas pedagógicas, y los profesores y los alumnos son los intérpretes que la desarrollan manifestando su estilo personal. (Gimeno, 1991: 3). Al respecto, Bolívar (2008) plantea que en el ámbito de la educación superior las competencias se convierten en los logros del aprendizaje, en lugar de la adquisición de conocimientos, afectando tanto a los objetivos, como al papel del profesorado, a las actividades de enseñanza y a la propia evaluación. Por ello, se puede decir que nos encontramos frente a un cambio paradigmático respecto al proceso de formación de profesionales. Desde esta perspectiva, se entiende el currículum como una realidad que expresa, por un lado, el problema de las relaciones entre la teoría y la práctica y, por otro, el de las relaciones entre la educación y la sociedad (Kemmis, 1988). En educación superior, plataformas como Moodle se presentan como idóneas para hacer tácita esa manera de concebirlo, ya que fomenta el aprendizaje colaborativo, la creatividad, la posibilidad de que los alumnos construyan a la par que el profesor y que posteriormente puedan aplicar lo construido y aprendido en su vida social y laboral (Ros, 2008).

Desde un punto de vista psicopedagógico, Moodle se configura en torno a lo que se denomina "pedagogía construccionista social" (Silva, 2011), es decir, "conjuga aspectos del constructivismo (el conocimiento se genera mediante mediación e interacción con el ambiente) y del construccionismo (aprender haciendo), además de que permite el aprendizaje colaborativo" (Sánchez, Sánchez y Ramos, 2012: 21). Presentándose como una de las principales herramientas, en el ejercicio de la docencia en el contexto universitario.

Siguiendo a Baumgartner (2005, citado en Sánchez, Sánchez y Ramos, 2012), esta herramienta, presenta tres tipos o modelos educativos de referencia:

a.Enseñanza I o de transmisión de conocimientos. En este modelo los aprendizajes de los estudiantes dependen, en gran medida, de los conocimientos del profesor y no hay una supervisión del proceso de aprendizaje. Tratándose de un modelo de enseñanza unidireccional.

b.Enseñanza II o de adquisición, compilación y acumulación de conocimientos. En este modelo se parte de la premisa de que la participación activa de los alumnos es una condición necesaria para el aprendizaje, en aras de que estos planifiquen, revisen y reflexionen sobre dicho conocimiento.

c. Enseñanza III o de desarrollo, invención y creación de conocimientos. El rol del profesor en este modelo es la de facilitador del aprendizaje, mientras los estudiantes son los que deben, a partir de la presentación de problemas del profesor, producir y generar su conocimiento. 
En base a esta tipología, nos situamos, entre el segundo y tercer modelo, ya que concebimos el proceso de enseñanza-aprendizaje en educación superior como un camino a construir con nuestros alumnos, que necesitan nuestro apoyo, orientaciones y guía de manera continua, ya que provienen de una serie de experiencias académicas donde presumiblemente hubo más enseñanza unidireccional que multidireccional. Si aceptamos que el currículo es una construcción cultural, debemos aceptar también que las creencias y experiencias de las personas involucradas en él, así como su manera de interactuar, determinan la forma y el tipo de sus prácticas educativas. (Agray, 2010: 423). Sin embargo, podemos observar que entre los profesores universitarios sigue estando muy instaurado el primer modelo, existiendo una distancia casi insalvable entre lo que se demanda a nivel social, teniendo en cuenta el contexto sociodigitalizado en el que nos desenvolvemos y el tipo de profesional que se está formando a partir de dicho modelo. Esta distancia entre el tipo de profesional que necesita la sociedad de la información y la forma en cómo están siendo preparados, es una situación que inevitablemente lleva a reflexionar sobre la pertinencia de los modelos curriculares que se están implementado, la efectividad de las metodologías de enseñanza y los modelos evaluativos que se están utilizando para el logro de las competencias que se requieren desarrollar (Roig y otros. 2013: 2).

Como afirman Gairín y Muñoz (2008), este cambio no es una necesidad, sino una realidad que se impone en nuestras sociedades dinámicas. Para ello, es necesario que instituciones educativas y especialmente profesionales de la educación formados, lo promuevan y lo canalicen. Este proceso de transformación requiere, según Salinas (2004), de cambios en cuatro importantes agentes: en el profesorado, en el alumnado, en las metodologías, y en las propias instituciones.

Tan cierto es el hecho de que el uso de las TIC en la docencia, no supone en sí mismo una innovación, como importante es resaltar que sin el uso de las mismas, difícilmente podemos abordar el reto de formar a los futuros profesionales en los diferentes ámbitos. En esta línea de reflexión, Järvelä (2006: 40) plantea una serie de principios como los mejores argumentos para la utilización de las TIC en el aprendizaje en términos potenciales, como son:

- Aumento del grado de autenticidad del aprendizaje y el interés del alumnado.

- Construcción de comunidades virtuales entre diferentes instituciones educativas, equipos colaborativos y profesorado.

- Ayuda para compartir perspectivas entre estudiantes con distintos bagajes, promoviendo la ayuda entre iguales y las prácticas de referencia en diferentes campos.

- Facilitación de la indagación mediada por la tecnología y los modelos de resolución de problemas para incrementar las habilidades de aprender a aprender. 
- Inclusión de formas innovadoras de integrar el apoyo sobre la marcha y las interacciones en diferentes contextos de aprendizaje.

Importante es resaltar el hecho de que muchos de los estudiantes universitarios poseen experiencias previas en el uso de una serie de herramientas tecnológicas, que hasta hace relativamente poco tiempo no eran de uso generalizado, lo que les posibilita unos aprendizajes que, con la ayuda de los docentes, tienen que poner al servicio de otros aprendizajes como futuros profesionales (más allá de los adquiridos en los ámbitos de ocio). Esto requiere acciones educativas relacionadas con el uso, selección, utilización y organización de la información, donde el apoyo y la orientación que recibirá en cada situación, así como la diferente disponibilidad tecnológica, son elementos cruciales en la explotación de las TIC, a través de actividades de formación significativas en el marco de este cambio de paradigma (Salinas, 2004).

Aunque no todos los estudiantes universitarios (de igual forma que ocurre con el profesorado) muestran una actitud positiva ante el uso de las TIC, como medio para trabajar las materias, existiendo una amplia variedad de tipos de alumnos, con diferentes inquietudes, expectativas, opiniones, actitudes..., apareciendo entre ellos los llamados "grupos tribus", quienes poseen una valoración bastante negativa sobre el uso de las TIC en su formación profesional (Gutiérrez, Palacios y Torrego, 2010).

En base a todo lo descrito, en esta experiencia investigativa, resulta especialmente interesante conocer el uso de las TIC que realizan los estudiantes, y de qué manera este interfiere en su comunicación social, en el uso y tratamiento que realizan de la información, en el desempeño y el aprendizaje adquiridos a través de las mismas en el contexto universitario (teniendo en cuenta la tarea mediadora del profesorado) y también, por supuesto, en la interacción social existente (con los demás miembros de la comunidad educativa), a través de diversas herramientas virtuales, en dicho contexto.

\section{METODOLOGÍA}

\subsection{Participantes}

La población y muestra del estudio estuvo compuesta por los 300 estudiantes pertenecientes al programa de Ciencias de la Actividad Física y el Deporte de la Corporación Universitaria del Caribe, CECAR (Sincelejo, Sucre, Colombia). El estudio fue realizado el mes de Noviembre de 2015, siendo la tasa de respuesta del $29,6 \%$ (89 alumnos). Suponiendo que la situación más desfavorable $(\mathrm{p}=\mathrm{q})$, y con un intervalo de confianza del $95 \%$, el error total de estimación fue $\pm 8,7 \%$. 


\subsection{Diseño y procedimiento}

El diseño del presente estudio es de tipo no experimental, al ser una investigación sistemática y empírica en la que las variables independientes no se manipulan porque ya han sucedido. Las inferencias sobre las relaciones entre variables se realizan sin intervención o influencia directa. Es también un diseño de tipo transversal descriptivo, al analizar el estado de diversas variables en un momento determinado; su propósito es describir variables y analizar su incidencia e interrelación en el momento del estudio (Sampieri, Collado y Lucio, 2003).

El cuestionario fue remitido a los correos electrónicos corporativos de los alumnos por parte de la sección de Comunicaciones de la Universidad en dos ocasiones (los días 6 y 20 de octubre de 2015), debido a que con el primer envío, la tasa de respuesta fue apenas del $9 \%$.

\subsection{Instrumento}

El instrumento utilizado en el presente estudio fue el cuestionario "Competencias básicas digitales 2.0 de estudiantes universitarios" COBADI 2013 (Zapata Ros, 2013). Se realizó un trabajo de adaptación del cuestionario en cuanto al español de Colombia y a las características de los estudiantes del Pregrado de Ciencias del Deporte. Para ello se llevaron a cabo diferentes etapas: Etapa 1. Evaluación gramatical, lingüística y semántica del cuestionario para adaptar el instrumento del español utilizado en España al español utilizado en Colombia. Etapa 2. Aceptabilidad y viabilidad del cuestionario. Mediante pilotaje a estudiantes de Ciencias del Deporte para ver la claridad con respecto a las preguntas planteadas. Etapa 3. Análisis validez del contenido. Pilotaje del cuestionario con docentes especialistas en el manejo de las tecnologías de la información y la comunicación). Etapa 4. Pilotaje del instrumento con muestra de estudiantes de Ciencias del Deporte.

Finalmente el instrumento estuvo formado por seis bloques y $18 \mathrm{items.} \mathrm{El} \mathrm{primero}$ de ellos tiene que ver con datos personales y el segundo, preguntas introductorias sobre el consumo de tecnología por parte de los alumnos. Los siguientes 4 bloques se dividen en: Bloque 1. Competencias en conocimiento y uso de las TIC en la comunicación social y aprendizaje colaborativo. Bloque 2. Competencias de uso de las TIC para la búsqueda y tratamiento de la información. Bloque 3. Competencias interpersonales en el uso de las TIC en el contexto universitario y Bloque 4. Herramientas virtuales y de comunicación social de la Universidad. Al finalizar se incluyó una pregunta abierta para poder recoger las opiniones de los alumnos con respecto a su experiencia en el uso de la plataforma Moodle durante el semestre en curso.

A través del mismo, este estudio se plantea contestar a una serie de cuestiones, tales como: 
- Si han recibido o no formación y el medio por el que han recibido formación los alumnos sobre la web 2.0?

- ¿Qué medios tecnológicos poseen? y ¿Dónde los usan?

- ¿Qué actividades realizan a través de Internet?

- ¿Cómo usan las TIC como medio social y como medio para aprender de manera colaborativa?

\subsection{Análisis de datos}

Se procedió a la tabulación y codificación de los cuestionarios y al volcado de estos datos en un paquete informático de análisis de datos PASW (Predictive Analytics SoftWare, v. 18.0 SPSS Inc., Chicago, IL, USA).

\section{RESULTADOS}

Participaron en el estudio 89 alumnos del Programa de Ciencias del Deporte y la Actividad Física, de los cuales 83 fueron hombres (93,3\%) y 6 mujeres $(6,7$ $\%$ ), con una edad media de $21,11 \pm 2,6$. De ellos, el $13,5 \%$ pertenecía al segundo semestre, $9 \%$ al tercer semestre, $5,6 \%$ al cuarto semestre, $13,5 \%$ al quinto semestre, $23,6 \%$ al sexto semestre, $4,5 \%$ al séptimo semestre, $16,9 \%$ al octavo semestre, $4,5 \%$ al noveno semestre y $9 \%$ al décimo semestre.

En el bloque relacionado con las preguntas sobre el consumo de tecnología por parte de los alumnos, en relación a si los alumnos habían recibido formación sobre web 2.0 o software social (blogs, redes sociales, correo electrónico, mensajería instantánea, Moodle, etc.), un elevado porcentaje de alumnos había recibido formación $(84,3 \%)$, frente a los que no la habían recibido $(15,7 \%)$ (Tabla 1$)$.

Tabla 1. Formación recibida por los alumnos sobre el uso de la web 2.0 o software social

\begin{tabular}{ccc}
\hline & $\mathrm{N}$ & Alumnos (\%) \\
\hline NO & 14 & 15,7 \\
SI & 75 & 84,3 \\
\hline & & Fuente: elaboración propia
\end{tabular}

Con respecto a los alumnos que habían recibido la formación sobre web 2.0 o software social, el mayor porcentaje correspondió a aquellos que habían recibido la formación en la Universidad (68\%), frente a los que su formación era autodidacta o había realizado cursos privados (Tabla 2). 
Tabla 2. Medio por el que fue recibida la formación sobre formación web 2.o o software social.

\begin{tabular}{lcc}
\hline & $\mathrm{N}$ & Alumnos (\%) \\
\hline Universidad & 61 & 68 \\
Autodidacta & 14 & 15,7 \\
Cursos privados & 5 & 5,6 \\
Otros & 9 & 1,1 \\
\hline
\end{tabular}

Con respecto a los medios tecnológicos que poseen los alumnos, un elevado porcentaje señaló que tenían ordenador propio, así como Internet en sus casas; aunque también puede observarse un elevado porcentaje entre aquellos alumnos que no tienen ordenador propio, además de aquellos que no tienen Internet en sus casas (Figura 1).

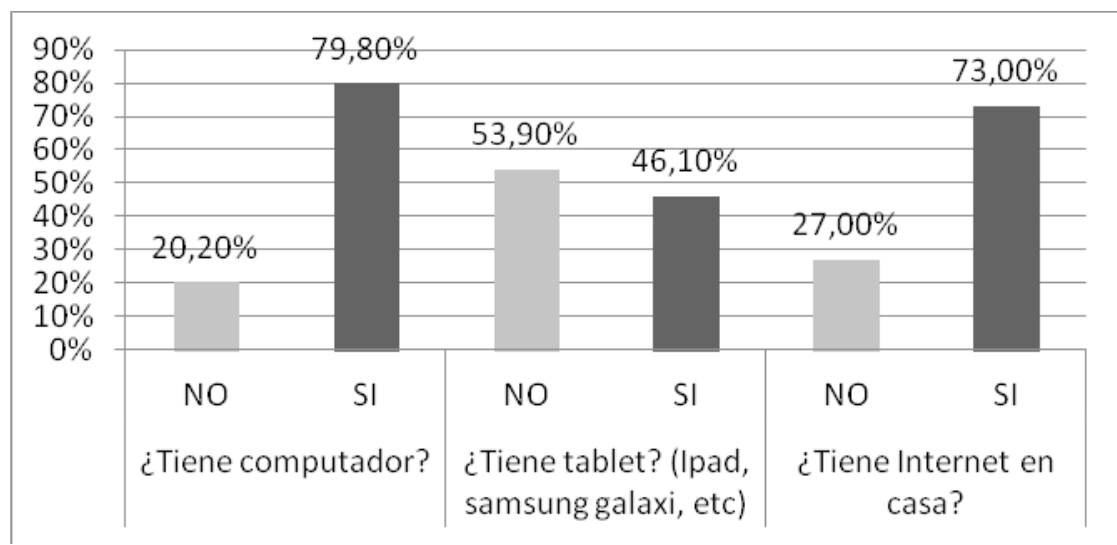

Figura 1. Medios tecnológicos que poseen los alumnos. Fuente: elaboración propia

Entre los lugares donde se conectan a Internet, comentar que en mayor proporción es en su casa, aunque también destacan la Universidad o en cualquier lugar, al tener Internet en el celular, como puede observarse a continuación (Tabla 3):

Tabla 3. Lugar de conexión habitual a Internet

\begin{tabular}{|l|c|c|}
\hline & $\mathrm{N}$ & Alumnos (\%) \\
\hline En casa & 53 & 59,6 \\
En casa de amigos & 4 & 4,5 \\
En cualquier sitio (dispongo de Internet en mi celular) & 14 & 15,7 \\
En la Universidad & 17 & 19,1 \\
En una tienda & 1 & 1,1 \\
\hline
\end{tabular}

Fuente: elaboración propia. 
En respuesta al tiempo que los alumnos pasan en Internet, significar que el $34,8 \%$ pasa entre 1 y 3 horas a la semana, siendo el mismo porcentaje que aquellos alumnos que pasan entre 4 y 9 horas a la semana. El 27\% pasan más de 9 horas a la semana, mientras que $1,1 \%$ restante pasa más de 48 horas a la semana navegando por la red.

En lo que se refiere al tiempo de uso de Internet en función de diferentes actividades, destaca el elevado porcentaje que lo utiliza, en mayor medida, para buscar información para tareas universitarias (78\%), realizar actividades académicas $(63 \%)$ y consultar información sobre temas académicos y profesionales.

Tabla 4. Actividades realizadas en internet

\begin{tabular}{|c|c|c|c|}
\hline & & $\mathrm{N}$ & Alumnos \% \\
\hline \multirow{3}{*}{ Ver programas de Televisión } & Mucho & 9 & 10,1 \\
\hline & Nada & 31 & 34,8 \\
\hline & Poco & 49 & 55,1 \\
\hline \multirow{3}{*}{ Escuchar música } & Mucho & 42 & 47,2 \\
\hline & Nada & 9 & 10,1 \\
\hline & Poco & 38 & 42,7 \\
\hline \multirow{3}{*}{$\begin{array}{l}\text { Información sobre temas académicos y } \\
\text { profesionales }\end{array}$} & Mucho & 61 & 68,5 \\
\hline & Nada & 1 & 1,1 \\
\hline & Poco & 27 & 30,3 \\
\hline \multirow{3}{*}{ Jugar online } & Mucho & 6 & 6,7 \\
\hline & Nada & 62 & 69,7 \\
\hline & Poco & 21 & 23,6 \\
\hline \multirow{3}{*}{ Buscar información para tareas universitarias } & Mucho & 78 & 87,6 \\
\hline & Nada & 11 & 12,4 \\
\hline & Poco & 0 & 0 \\
\hline \multirow{3}{*}{ Publicar fotografías/ vídeos } & Mucho & 11 & 12,4 \\
\hline & Nada & 18 & 20,2 \\
\hline & Poco & 60 & 67,4 \\
\hline \multirow[t]{3}{*}{ Descargar música, películas, juegos, etc. } & Mucho & 15 & 16,9 \\
\hline & Nada & 25 & 28,1 \\
\hline & Poco & 49 & 55,1 \\
\hline \multirow{3}{*}{$\begin{array}{l}\text { Hablar con los amigos a través de redes } \\
\text { sociales, chat, etc. }\end{array}$} & Mucho & 39 & 43,8 \\
\hline & Nada & 1 & 1,1 \\
\hline & Poco & 49 & 55,1 \\
\hline \multirow{3}{*}{$\begin{array}{l}\text { Buscar amigos/as nuevos mediante el uso de } \\
\text { redes sociales }\end{array}$} & Mucho & 20 & 22,5 \\
\hline & Nada & 26 & 29,2 \\
\hline & Poco & 43 & 48,3 \\
\hline \multirow[t]{3}{*}{ Realizar tareas académicas } & Mucho & 63 & 70,8 \\
\hline & Nada & 1 & 1,1 \\
\hline & Poco & 25 & 28,1 \\
\hline
\end{tabular}

Fuente: elaboración propia. 
Con respecto a las competencias y uso de las TIC en la comunicación social y el aprendizaje colaborativo, en una escala de 1 a 4 , en la que el 1 hace referencia a lo ineficaz que se siente el alumno y el 4 hace referencia al dominio completo sobre el conocimiento y uso de la herramienta planteada. Un elevado número de alumnos afirman encontrarse en el nivel de dominio completo (4): "me puedo comunicar con otras personas mediante correo electrónico" (66,3\%), "utilizo el chat para relacionarme" $(52,8 \%)$, "uso la mensajería instantánea como herramienta de comunicación" $(49,4 \%)$, "puedo comunicarme con otras personas participando en redes sociales" $(56,2 \%)$, "soy capaz de desenvolverme en redes de ámbito profesional" (29,2\%), "soy capaz de participar de modo apropiado en foros"( $41,6 \%)$, "sé utilizar las Wikis" (37,1\%), "soy capaz de utilizar plataformas educativas, (WebCt, campus on line, intranet, Moodle, Dokeos, etc)" (57,3\%).

Tabla 5. Competencias y uso de las TIC en la comunicación social y el aprendizaje colaborativo

\begin{tabular}{|c|c|c|c|}
\hline & & $\mathrm{N}$ & $\begin{array}{c}\text { Alumnos } \\
\%\end{array}$ \\
\hline \multirow[t]{5}{*}{ Me puedo comunicar con otras personas mediante correo electrónico } & 1 & 3 & 3,4 \\
\hline & 2 & 4 & 4,5 \\
\hline & 3 & 22 & 24,7 \\
\hline & 4 & 59 & 66,3 \\
\hline & 5 & 1 & 1,1 \\
\hline \multirow[t]{5}{*}{ Utilizo el Chat para relacionarme con otras personas. } & 1 & 4 & 4,5 \\
\hline & 2 & 11 & 12,4 \\
\hline & 3 & 26 & 29,2 \\
\hline & 4 & 47 & 52,8 \\
\hline & 5 & 1 & 1,1 \\
\hline \multirow{5}{*}{$\begin{array}{l}\text { Uso la mensajería instantánea como herramienta de comunicación con } \\
\text { otras personas. }\end{array}$} & 1 & 5 & 5,6 \\
\hline & 2 & 8 & 9,0 \\
\hline & 3 & 29 & 32,6 \\
\hline & 4 & 44 & 49,4 \\
\hline & 5 & 3 & 3,4 \\
\hline \multirow{5}{*}{$\begin{array}{l}\text { Puedo comunicarme con otras personas participando en redes sociales } \\
\text { (ning, facebook, twitter, hi5, myspace, tuenti, etc). }\end{array}$} & 1 & 5 & 5,6 \\
\hline & 2 & 10 & 11,2 \\
\hline & 3 & 23 & 25,8 \\
\hline & 4 & 50 & 56,2 \\
\hline & 5 & 1 & 1,1 \\
\hline \multirow{5}{*}{$\begin{array}{l}\text { Soy capaz de desenvolverme en redes de ámbito profesional (linked } \\
\text { in, xing). }\end{array}$} & 1 & 10 & 11,2 \\
\hline & 2 & 16 & 18,0 \\
\hline & 3 & 24 & 27,0 \\
\hline & 4 & 26 & 29,2 \\
\hline & 5 & 13 & 14,6 \\
\hline \multirow[t]{5}{*}{ Soy capaz de participar de modo apropiado en foros. } & 1 & 3 & 3,4 \\
\hline & 2 & 20 & 22,5 \\
\hline & 3 & 28 & 31,5 \\
\hline & 4 & 37 & 41,6 \\
\hline & 5 & 1 & 1,1 \\
\hline
\end{tabular}




\begin{tabular}{|c|c|c|c|}
\hline \multirow[t]{5}{*}{ Me considero competente para participar en blogs. } & 1 & 5 & 5,6 \\
\hline & 2 & 19 & 21,3 \\
\hline & 3 & 34 & 38,2 \\
\hline & 4 & 26 & 29,2 \\
\hline & 5 & 5 & 5,6 \\
\hline \multirow{5}{*}{$\begin{array}{l}\text { Sé diseñar, crear y modificar Blogs o bitácoras (por ejemplo: blogger, } \\
\text { wordpress, etc.). }\end{array}$} & 1 & 13 & 14,6 \\
\hline & 2 & 32 & 36,0 \\
\hline & 3 & 24 & 27,0 \\
\hline & 4 & 11 & 12,4 \\
\hline & 5 & 9 & 10,1 \\
\hline \multirow[t]{5}{*}{ Sé utilizar las Wikis (wikipedia, aulawiki21, etc). } & 1 & 9 & 10,1 \\
\hline & 2 & 17 & 19,1 \\
\hline & 3 & 25 & 28,1 \\
\hline & 4 & 33 & 37,1 \\
\hline & 5 & 5 & 5,6 \\
\hline \multirow{5}{*}{$\begin{array}{l}\text { Me considero competente para diseñar, crear o modificar una } \\
\text { wiki (wikispace, nirewiki, PbWorks..., etc). }\end{array}$} & 1 & 21 & 23,6 \\
\hline & 2 & 21 & 23,6 \\
\hline & 3 & 21 & 23,6 \\
\hline & 4 & 16 & 18,0 \\
\hline & 5 & 10 & 11,2 \\
\hline \multirow[t]{5}{*}{ Uso el sistema de sindicación (RSS). } & 1 & 20 & 22,5 \\
\hline & 2 & 19 & 21,3 \\
\hline & 3 & 14 & 15,7 \\
\hline & 4 & 4 & 4,5 \\
\hline & 5 & 32 & 36,0 \\
\hline \multirow{5}{*}{$\begin{array}{l}\text { Sé usar los marcadores sociales, etiquetado, "social } \\
\text { bookmarking" (delicious, blinklist,... }\end{array}$} & 1 & 20 & 22,5 \\
\hline & 2 & 22 & 24,7 \\
\hline & 3 & 15 & 16,9 \\
\hline & 4 & 13 & 14,6 \\
\hline & 5 & 19 & 21,3 \\
\hline \multirow{5}{*}{$\begin{array}{l}\text { Soy capaz de utilizar plataformas educativas, (WebCt, } \\
\text { campus on line, intranet, Moodle, Dokeos, etc). }\end{array}$} & 1 & 7 & 7,9 \\
\hline & 2 & 10 & 11,2 \\
\hline & 3 & 17 & 19,1 \\
\hline & 4 & 51 & 57,3 \\
\hline & 5 & 4 & 4,5 \\
\hline
\end{tabular}

Fuente: elaboración propia.

En relación con las competencias de uso de las TICs para la búsqueda y tratamiento de la información, utilizando la escala anteriormente citada del 1 al 4, destacan aquellas competencias en las que un elevado porcentaje de alumnos afirman que poseen un nivel de dominio completo (4 en la escala). Estos son "navegar por Internet con diferentes navegadores (Mozilla, Opera, Explorer, Chrome, etc.)" (70,8\%), capaz de usar distintos buscadores (Google, Ixquick, Mashpedia, etc.) 
(65,2\%), sé usar programas para planificar mi tiempo de estudio (google calendar...) $(36 \%)$.

En referencia a herramientas virtuales y de comunicación social de la Universidad, destacar que el 83,1\% de los encuestados, afirma dominar completamente el uso de la plataforma virtual Moodle de la Universidad, mientras que el 71,9\% afirma dominar completamente el correo electrónico de la Universidad.

\section{DISGUSIÓN Y CONGLUSIONES}

Esta investigación se presenta como un estudio analítico a tenor de las características de la Sociedad del Siglo XXI, sin perder de vista los cambios continuos y el manejo de las Tecnologías de la Información y la Comunicación que la configuran como una etapa idiosincrásica en sí misma. Por tanto, es evidente la necesidad de conocer de dónde se parte en el manejo de las TIC por parte del alumnado, para poder fortalecer un aprendizaje permanente, autónomo y crítico a lo largo de toda la vida, que permita a los mismos desenvolverse de manera eficaz en las diferentes situaciones que se le presenten en su transcurrir diario.

Al igual que en el estudio de Veytia (2013), los resultados que presenta esta investigación permiten valorar las fortalezas y áreas de oportunidad de los estudiantes (en este caso de Pregrado) y su dominio en cuanto a la competencia digital, a partir de caracterizar la población objeto de estudio, 89 alumnos del Programa de Ciencias del Deporte y la Actividad Físca, de los cuales 83 fueron hombres $(93,3 \%)$ y 6 mujeres $(6,7 \%)$, con una edad promedio de 21,11 años.

Coincidiendo con Veytia (2013), entre los principales hallazgos de este estudio, se encuentra en que una gran mayoría de alumnos se consideran muy capaces de utilizar, de manera eficiente, herramientas tales como: diferentes navegadores, correo electrónico, plataforma Moodle..., a través de las cuales desarrollan su competencia social en la Universidad.

A diferencia del estudio de Roig y otros (2013), en este estudio se extrae que el perfil de los estudiantes encuestados se enmarca dentro de personas que se conectan entre 1 y 9 horas, mayoritariamente, a la semana a Internet . Coincidiendo con estos autores en que utilizan, de manera más acentuada, este recurso para la realización de búsquedas con el fin de llevar a cabo trabajos relacionados con las tareas de la Universidad.

La principal limitación del estudio, radica en el hecho de que estos datos son meramente informativos y no han sido utilizados, hasta el momento, como punto de partida, por ejemplo, en la construcción de un programa de evaluación que mida, desde la propia práctica académica, el nivel de competencia digital tanto de alumnos como de maestros y reorientar la práctica formativo-académica a tenor de los resultados que se pudieran obtener. También sería interesante realizar un estudio 
longitudinal aumentando la muestra y realizando una comparativa entre universidades de distintos países, con alumnos que pertenezcan a parecidos y/o desiguales niveles socioeconómicos, lo cual posibilitaría analizar desde una perspectiva más amplia los factores que se miden en el cuestionario utilizado para el estudio.

Estos resultados hacen evidente que si bien el grupo de estudiantes maneja las Tecnologías de la Información y Comunicación, es fundamental seguirlas fortaleciendo y desarrollando a través de la práctica académica, posibilitando la mejora de educadores y educandos en su uso y manejo y favoreciendo una mayor profesionalidad de los segundos, cuando estos se incorporen al ámbito laboral. Esto refuerza la continuación de realizar esfuerzos por investigar las implicaciones organizativas, metodológicas y de evaluación de las TIC en el desempeño de la docencia universitaria (Sánchez, Sánchez y Ramos, 2012).

Igualmente, estos resultados, pese a sus limitaciones y a las cautelas necesarias por las características del estudio, nos ofrecen claves para seguir mejorando la formación que ofrecemos en nuestras facultades. Para seguir mejorando la formación de los futuros profesionales y, con ello, hacer posible la construcción de una sociedad adaptada a las nuevas demandas educativo-formativas (Esteve Mon, 2015).

\section{REFERENGIAS BIBLIOGRÁFICAS}

Blázquez, F. (2001). Sociedad de la información y educación. Mérida: Consejería de Educación, Ciencia y Tecnología.

Cole, J. R. y Helen, C. (2007). Using Moodle: Teaching With the Popular Open Source Course Management System. Nueva York: O'Reilly Media, Inc.

Escamilla, A. (2008) Las Competencias Básicas. Claves y propuestas para su desarrollo en los Centros. Barcelona: Gráo.

Gimeno, J. (1991). El currículum: una reflexión sobre la práctica. Madrid: Morata.

Gutiérrez, A., Palacios, A. y Torrego, L. (2010). Tribus digitales en las aulas universitarias. Comunicar, 34, 173-181. Doi:10.3916/C34-2010-03-17

Kemmis, S. (1988). El currículum: más allá de la teoría de la reproducción. Madrid: Morata.

Palomo, R., Ruiz, J. y Sánchez, J. (2006). Las TIC como agentes de innovación educativa. Sevilla: Consejería de Educación.

Pérez Rodríguez, M. T. y otros (2009). Innovación en docencia universitaria con Moodle: casos prácticos. San Vicente del Raspeig: Club Universitario.

Sampieri, R., Collado, C. y Lucio: (2003). Metodología de la investigación. McGrawHill.

Silva, J. (2011). Diseño y moderación de entornos virtuales de aprendizaje (eva). Barcelona: UOC. 


\section{FUENTES ELEGTRÓNICAS}

Abad, M. R. (2005). El compromiso de los estados a partir de la cumbre mundial sobre la sociedad de la información. Telos: Cuadernos De Comunicación, Tecnología y Sociedad, 63, 100-109. Consultado el 10 de Septiembre de 2015, desde https:// telos.fundaciontelefonica.com/telos/articuloderecho.asp@idarticulo=1\&rev=63. htm

Agray, N. (2010). La construcción de currículo desde perspectivas críticas: una producción cultural. Signo y pensamiento, 56, 420-427. Consultado el 15 de Septiembre de 2015, desde http://revistas.javeriana.edu.co/index.php/signoypensamiento/article/ download/2571/1839.

Almerich, G. y otros (2005). Diferencias en los conocimientos de los recursos tecnológicos en profesores a partir del género, edad y tipo de centro. Revista Electrónica de Investigación y Evaluación Educativa, 11(2). Consultado el 26 de Noviembre de 2015, desde http://www.uv.es/relieve/v11n2/RELIEVEv11n2_3.htm

Area, M. (2010). ¿Por qué formar en competencias informacionales y digitales en la educación superior? Revista de Universidad y Sociedad del Conocimiento, 7(2), 2-4. Consultado el 20 de Octubre de 2015, desde http://rusc.uoc.edu/index.php/ rusc/article/viewFile/v7n2-area/v7n2-competencias-informacionales-y-digitalesen-educacion-superior

Blasco A. (2005) Ideas para la construcción del Espacio Iberoamericano de Educación Superior. Revista de Ciencias Sociales del IUIOG, 8, 1-12. Consultado el 20 de Octubre de 2015, desde http://www.ortegaygasset.edu/publicaciones/circunstancia/ ano-iii---numero-8---septiembre-2005/ensayos/ideas-para-la-construccion-delespacio-iberoamericano-de-educacion-superior

Bolívar, A. (2008). El discurso de las competencias en España: educación básica y educación superior. Revista de Docencia Universitaria, 6(2). Consultado el 1 de Noviembre de 2015, desde http://redaberta.usc.es/redu/index.php/REDU/article/ view/70

Cabero, J. y Llorente, M. C. (2005). Las plataformas virtuales en el ámbito de la teleformación, Revista electrónica Alternativas de educación y comunicación. Consultado el 18 de Octubre de 2015, desde http://tecnologiaedu.us.es/cuestionario/ bibliovir/plataformas_virtuales_teleformacion_2005.pdf

Cabero, J. y Llorente, M.C. (2008). La alfabetización digital de los alumnos. Competencias Digitales para el siglo XXI. Revista Portuguesa de Pedagogía, 42(2), 7-28. Consultado el 20 de Octubre de 2015, desde http://tecnologiaedu.us.es/ cuestionario/bibliovir/jca26.pdf

De Pablos, J. (2010). Universidad y sociedad del conocimiento. Las competencias informacionales y digitales. Revista de Universidad y Sociedad del Conocimiento, 7 (2), 6-15. Consultado el 17 de Septiembre de 2015, desde http://rusc.uoc.edu/ index.php/rusc/article/viewFile/v7n2-area/v7n2-competencias-informacionales-ydigitales-en-educacion-superior 
Esteve Mon, F. M. y Gisbert, M. (2011). El nuevo paradigma de aprendizaje y las nuevas tecnologías. Revista de Docencia Universitaria, 9(3), 55-73. Consultado el 15 de Septiembre de 2015, desde http://red-u.net/redu/files/journals/1/articles/301/ public/301-626-1-PB.pdf

Esteve Mon, F. M. (2015). La competencia digital docente. Análisis de la autopercepción y evaluación del desempeño de los estudiantes universitarios de educación por medio de un entorno 3D. Consultado el 26 de Noviembre de 2015, desde http://www.tdx.cat/bitstream/handle/10803/291441/tesis. pdf;jsessionid=C5E7D08AECAFA97892E3B542F91FA019.tdx1? sequence $=1$

Gairín, J., y Muñoz, J. L. (2008). El agente de cambio en el desarrollo de las organizaciones. Enseñanza \& Teaching, 26, 187-206. Consultado el 26 de Noviembre de 2015, desde http://gredos.usal.es/jspui/bitstream/10366/69667/1/El_agente_de_cambio_en_el_ desarrollo_de_pdf

Järvelä, S. (2006). Personalised Learning? New Insights into Fostering Learning Capacity. En OCDE-CERI (eds.), Personalising Education (pp. 31-46). Paris: OCDE/ CERI. Consultado el 2 de Noviembre de 2015, desde http:/www.oecd.org/site/ schoolingfortomorrowknowledgebase/themes/demand/41176687.pdf

Marín, V., Ramírez, A., y Sampedro, B. (2011). Moodle y estudiantes universitarios. Dos nuevas realidades del EEES Profesorado. Revista de Currículum y Formación de Profesorado, 15(1), 109-120. Consultado el 17 de abril de 2017 de www.redalyc.org/ pdf/567/56717469008.pdf

Prensky, M. (2001). Digital Natives, Digital Immigrants. On the Horizon, 9(5), 1-6. Consultado el 30 de Agosto de 2015, desde http://www.marcprensky.com/writing/ Prensky\%20-\%20Digital\%20Natives,\%20Digital\%20Immigrants\%20-\%20Part1. pdf

Rodríguez Damián, A. y otros (2009). Las TIC en la educacion superior: estudio de los factores intervinientes en la adopción de un LMS por docentes innovadores. RELATEC, Revista Latinoamericana de Tecnología Educativa, 8(1), 35-51. Consultado el 15 de Octubre de 2015, desde http://dialnet.unirioja.es/servlet/articulo? codigo=3039592

Roig, R. y otros (2013). Evaluación de las Competencias Digitales del alumnado en el Espacio Europeo de Educación Superior (Proyecto “e-Accesible”). Consultado el 29 de Octubre de 2015, desde http://web.ua.es/es/ice/jornadas-redes-2012/documentos/ posters/243944.pdf

Ros, I. (2008). Moodle, la plataforma para la enseñanza y organización escolar. Ikastorratza, e-Revista de Didáctica, 2, 1-12. Consultado el 1 de Octubre de 2015, desde http:// www.ehu.es/ikastorratza/2_alea/moodle.pdf

Salinas, J. (2004). Innovación y uso de las TIC en la enseñanza universitaria. Revista Universidad y Sociedad del Conocimiento, 1(1), 1-16. Consultado el 3 de Noviembre, desde http://www.uoc.edu/rusc/dt/esp/salinas1104.pdf

Sánchez, J., Sánchez: y Ramos, F. J. (2012). Usos pedagógicos de Moodle en la docencia universitaria desde la perspectiva de los estudiantes. Revista Iberoamericana de 
Educación, 60, 15-38. Consultado el 5 de Septiembre de 2015, desde http://www. rieoei.org/rie60a01.pdf

Veytia, M. G. (2013). Competencias básicas digitales en estudiantes de postgrado. Revista Electrónica de Investigación en Educación Superior, 13, 1-10. Consultado el 25 de Septiembre de 2015, desdehttp://www.iiies.org/reedies/index.php?html=27

Zapata Ross, M. (2013) Competencias Básicas Digitales 2.0 de los estudiantes universitarios COBADI 2013 (marca registrada: 2970648). Consultado el 1 de 1 de Septiembre de 2015, desde: http://www.scoop.it/t/competencia-digital-docente 